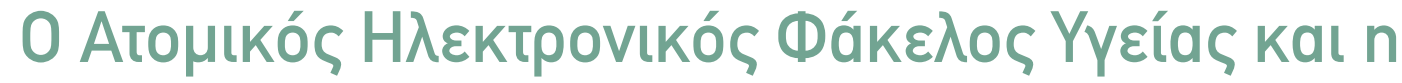

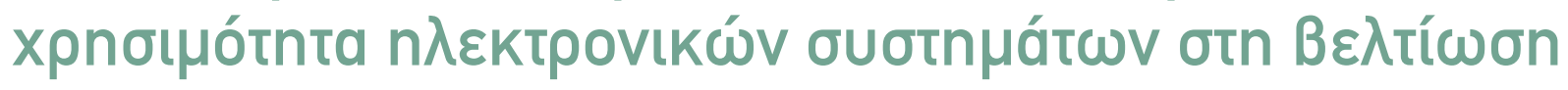

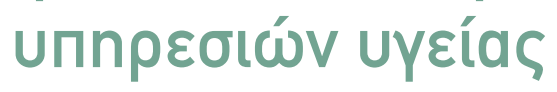

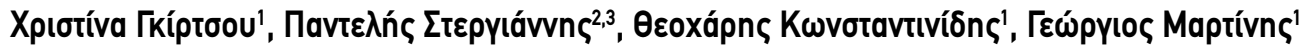

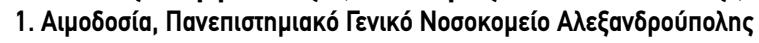

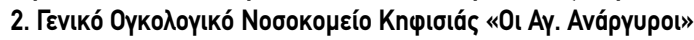

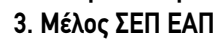

\section{ПЕРIАHШH}

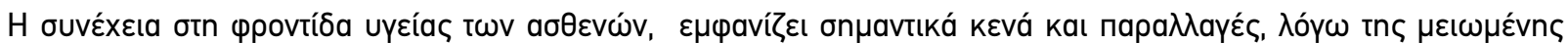

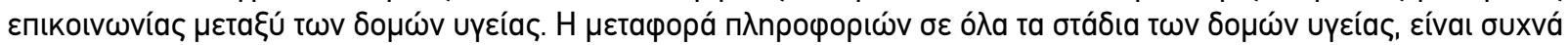

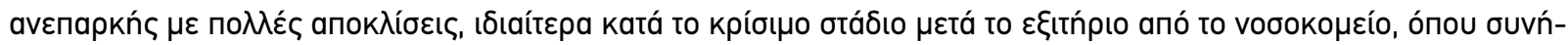

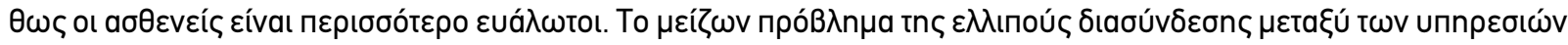

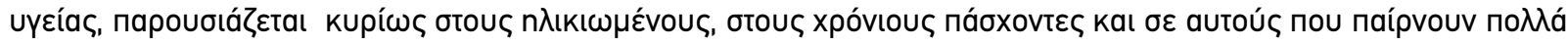

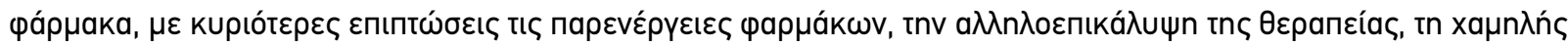

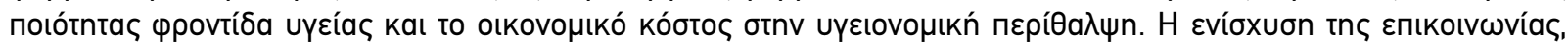

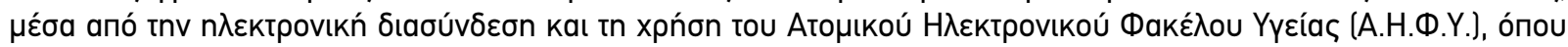

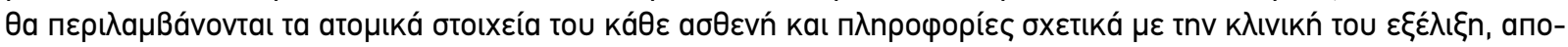

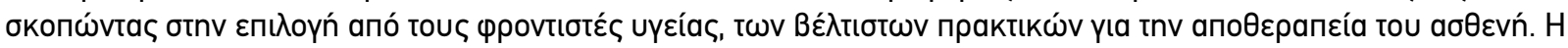

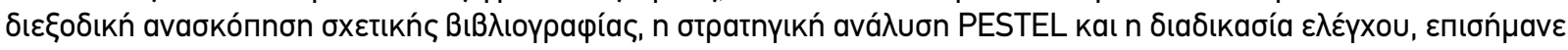

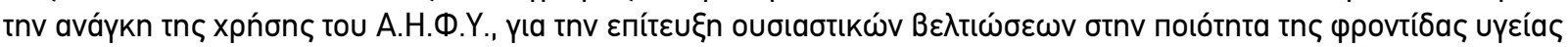

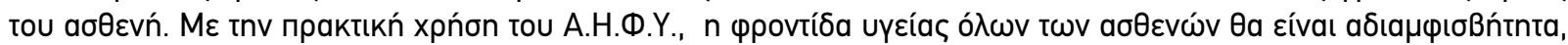

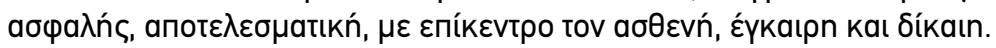

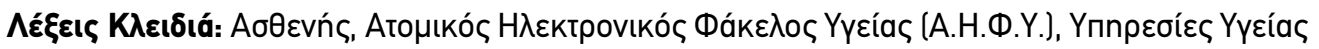




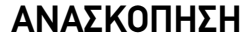

\section{EIIAГОГН}

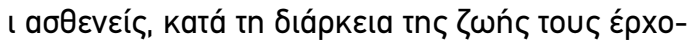

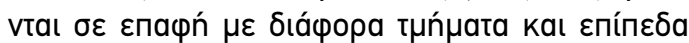

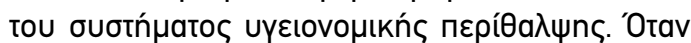

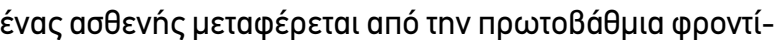

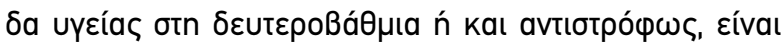

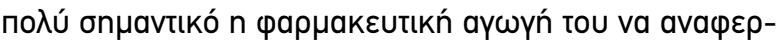

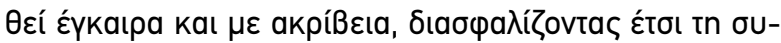

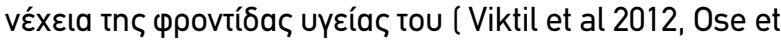

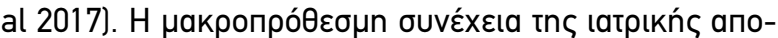

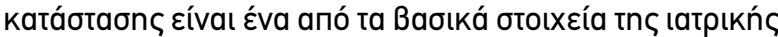

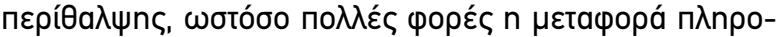

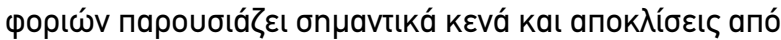

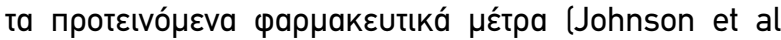

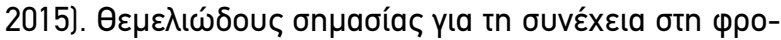

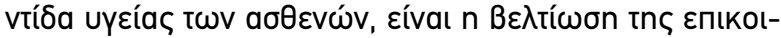

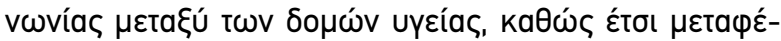

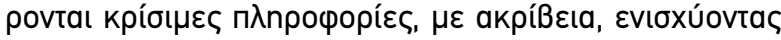

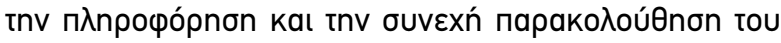

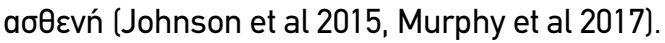

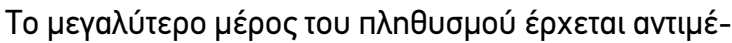

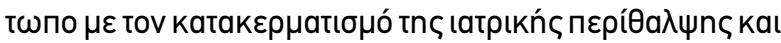

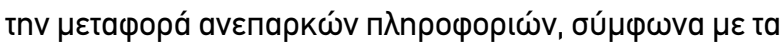

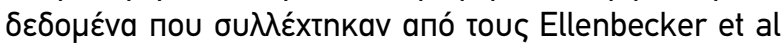

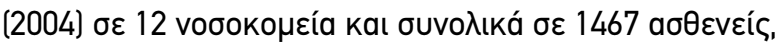

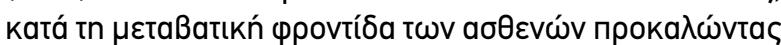

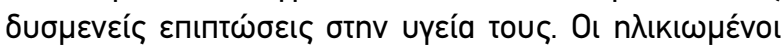

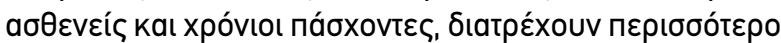

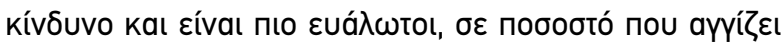
to $40 \%$, $\delta \varepsilon \delta$

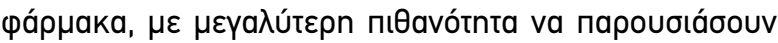

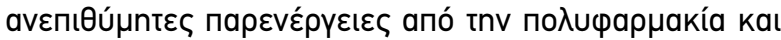

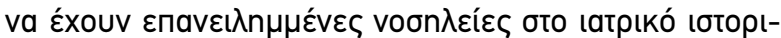

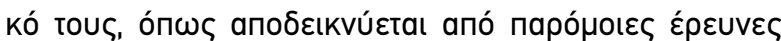
(Coleman et al 2003, Midlöv et al 2008, LaMantia et al 2010, Salvi et al 2012, Skoog et al 2015, Tiihonen et al 2016, Caleres et al 2018). Oı Salvi et al (2012), otn $\mu \varepsilon-$

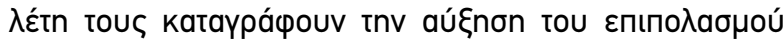

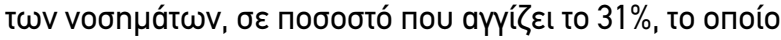

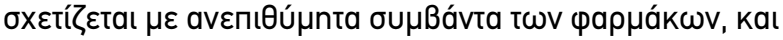

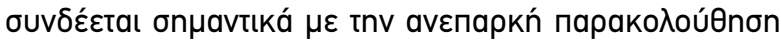

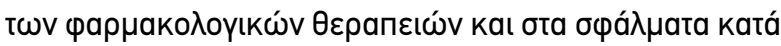

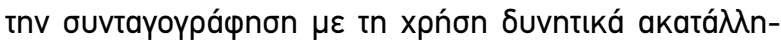

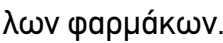

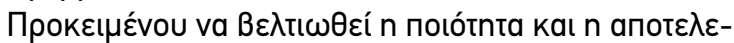

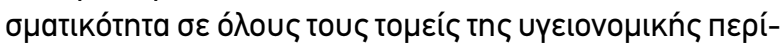

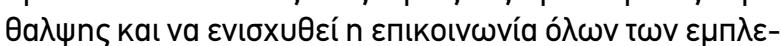

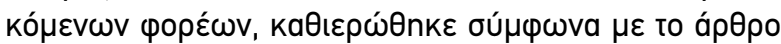

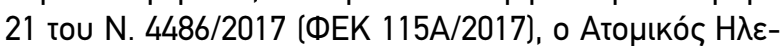

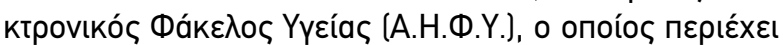

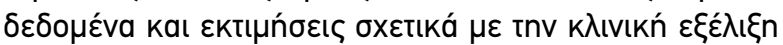

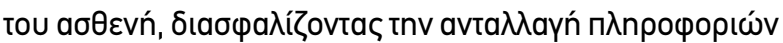

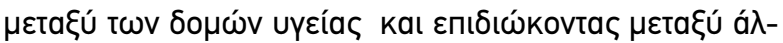

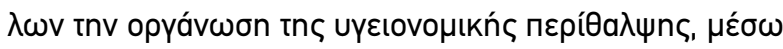

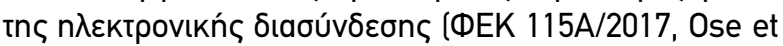
al 2017).

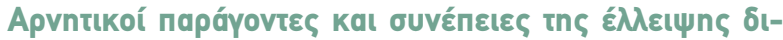

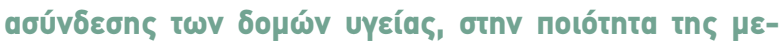

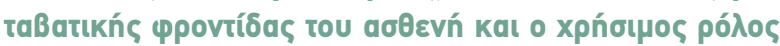
Tou A.H.Ф.Y.

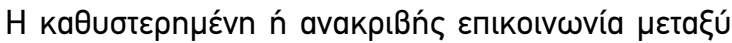

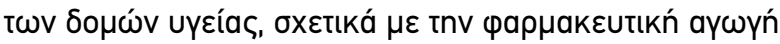

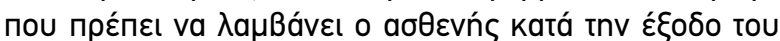

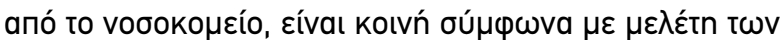

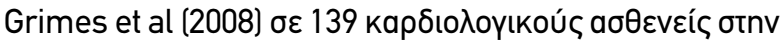

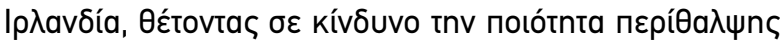

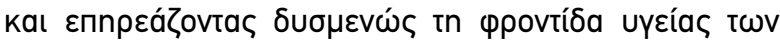

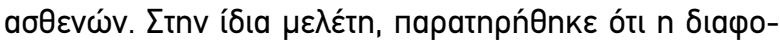

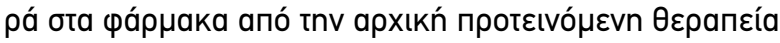

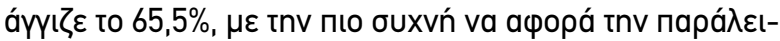

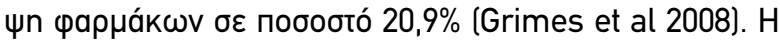

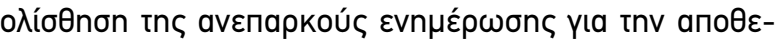

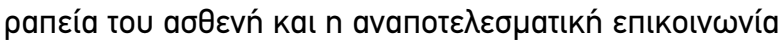

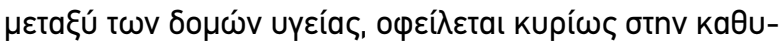

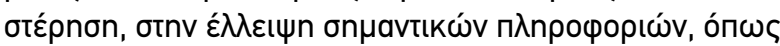

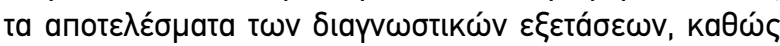

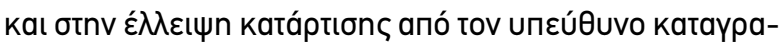
фńc (Belleli et al 2013).

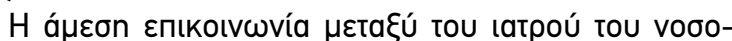

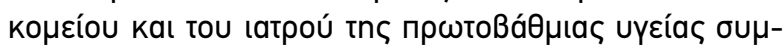

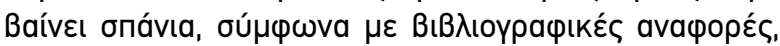

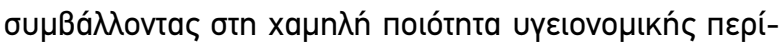

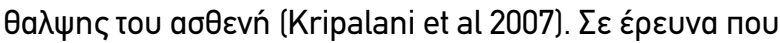

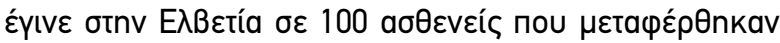

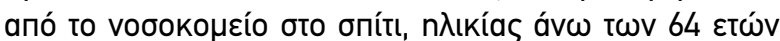

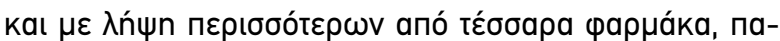

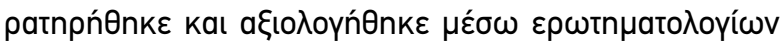

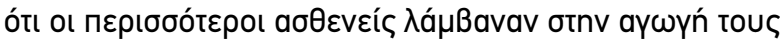

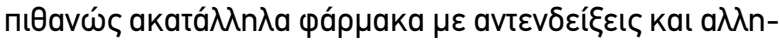

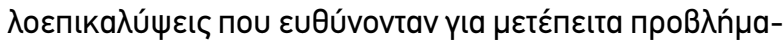

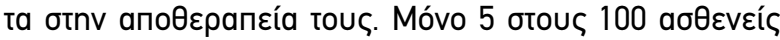

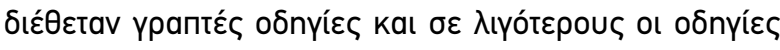

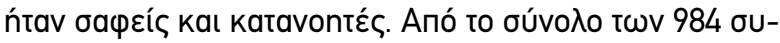

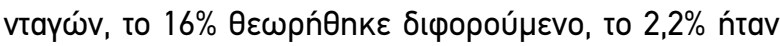

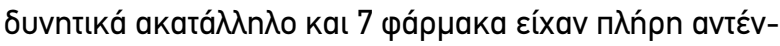
$\delta \varepsilon ı \xi n$ (Meyer-Massetti et al 2018).

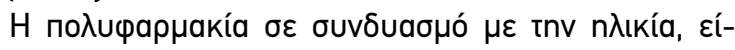

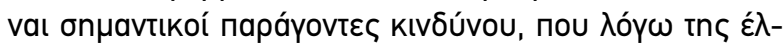

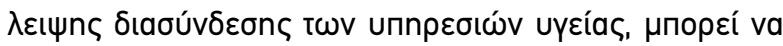

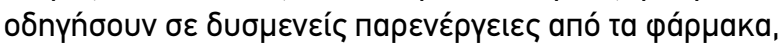

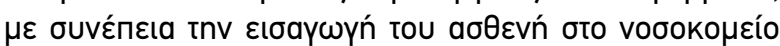

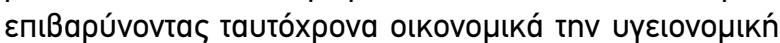

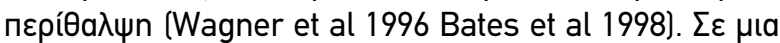

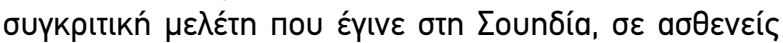

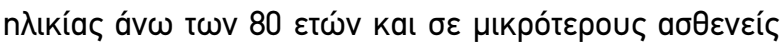




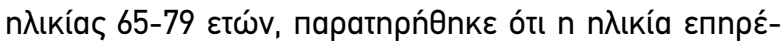

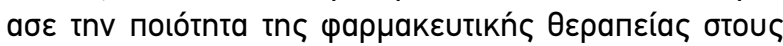

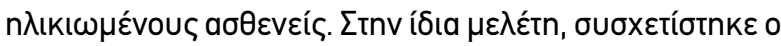

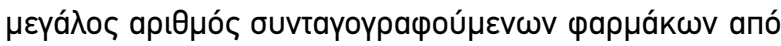

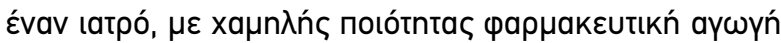

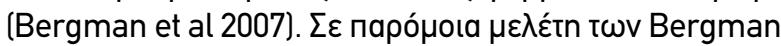

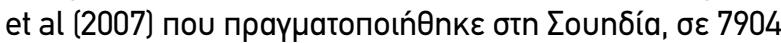

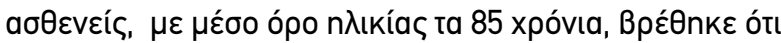

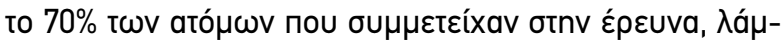

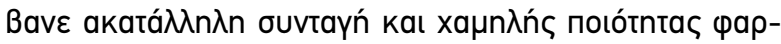

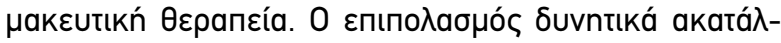

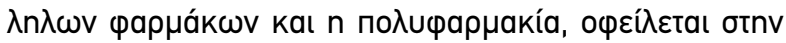

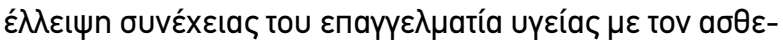

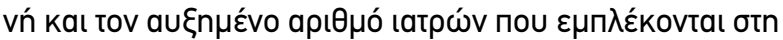

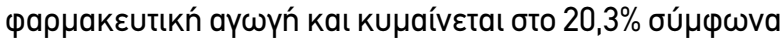

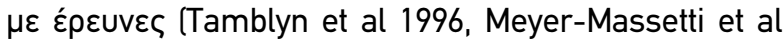
2012).

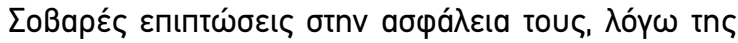

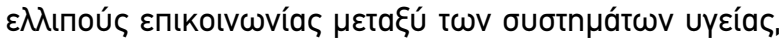

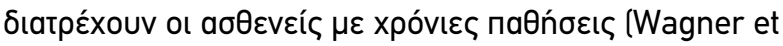

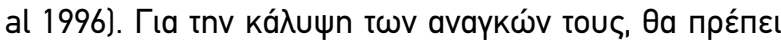

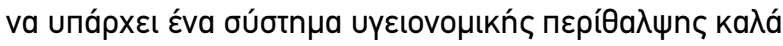

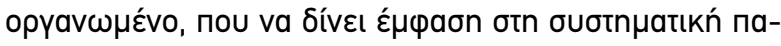

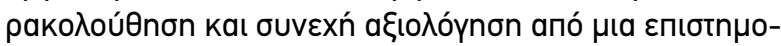

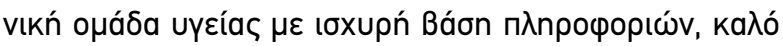

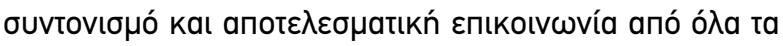

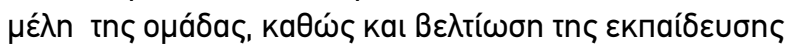

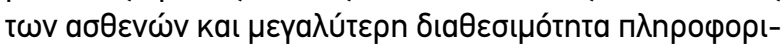
$\omega ́ v$ (Wagner et al 1996, Ose et al 2017, Brown et al 2018).

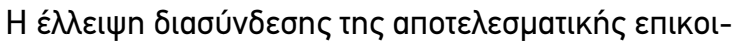

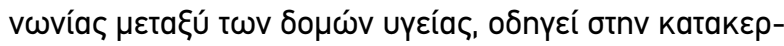

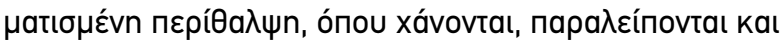

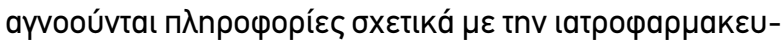

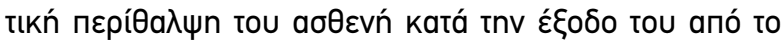

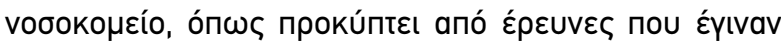

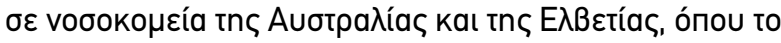

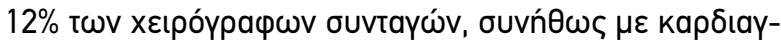

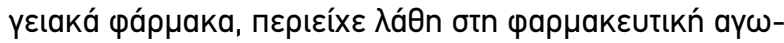

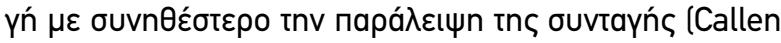
et al 2010, Abdel-Qader et al 2011, Belleli et al 2013).

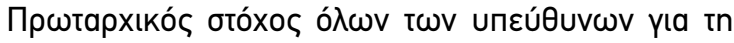

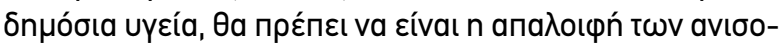

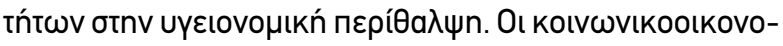

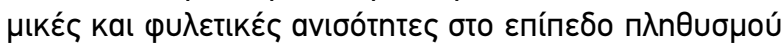

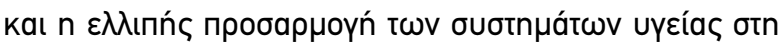

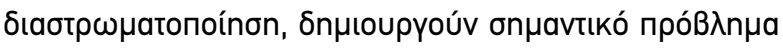

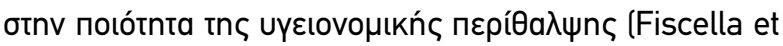
al 2000).

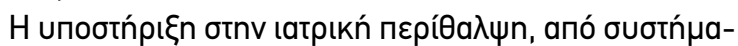

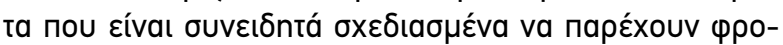

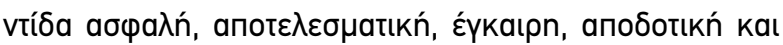

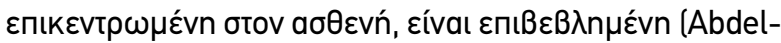

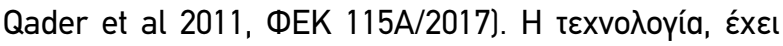

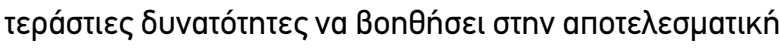

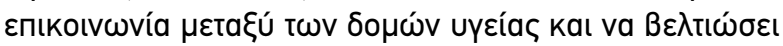

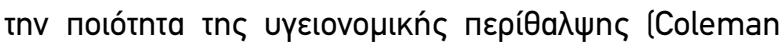

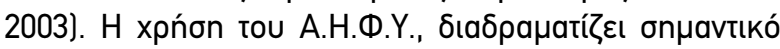

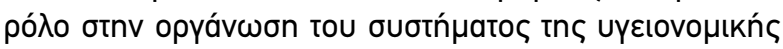

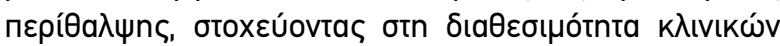

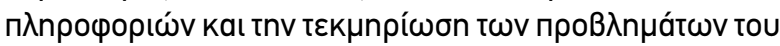

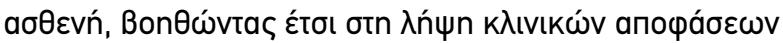

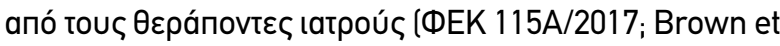

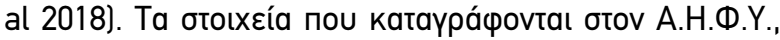

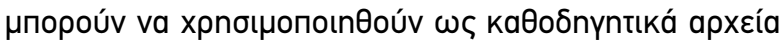

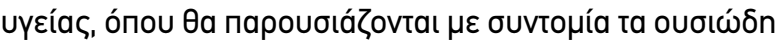

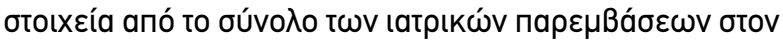

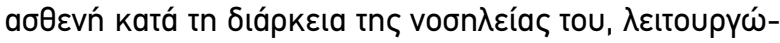

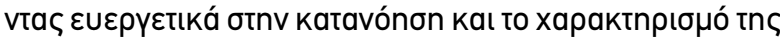

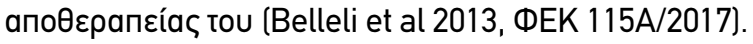

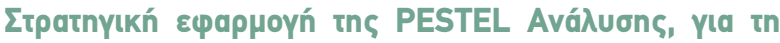

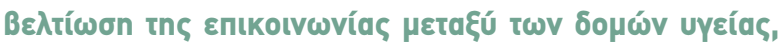
$\mu \varepsilon$ tn Xpńon tou A.H.Ф.Y.

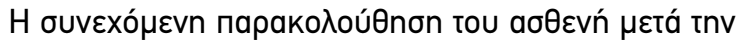

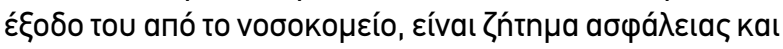

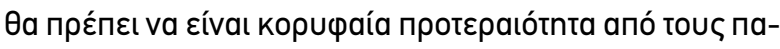

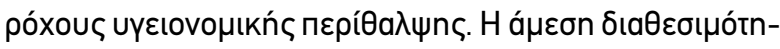

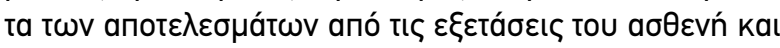

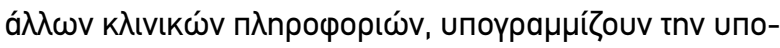

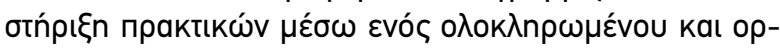

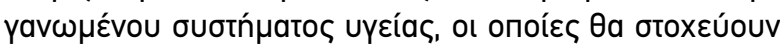

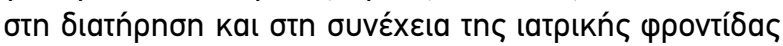
(Gandhi 2005, Chen et al 2010, Brown et al 2018).

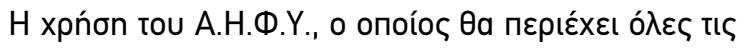

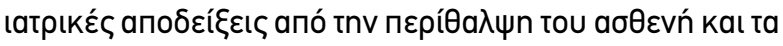

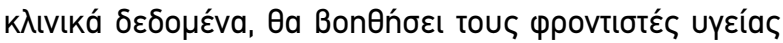

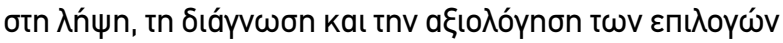

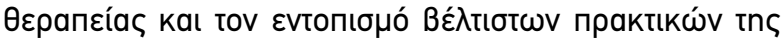

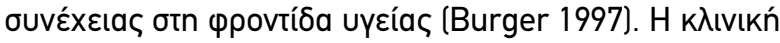

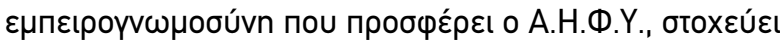

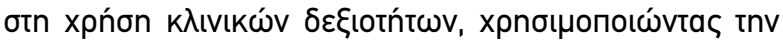

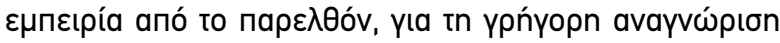

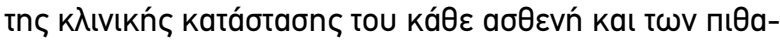

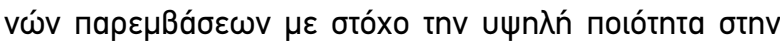

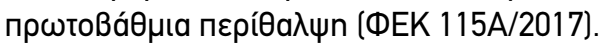

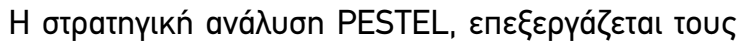

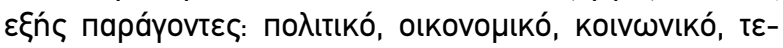

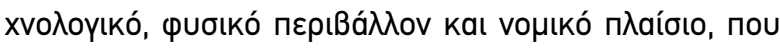

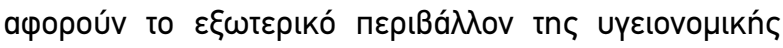

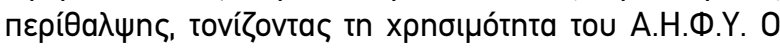

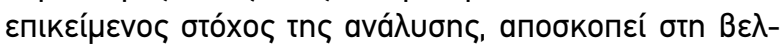

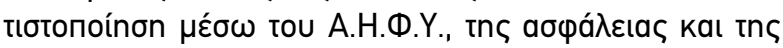

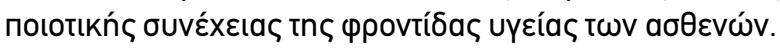

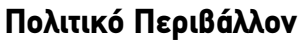

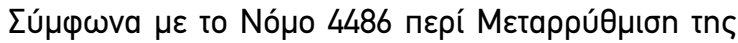




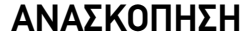

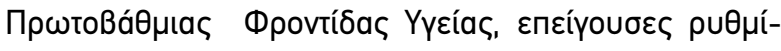

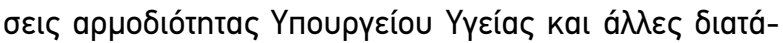

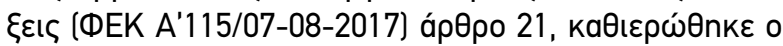

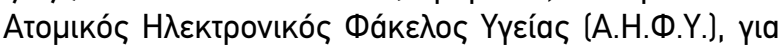

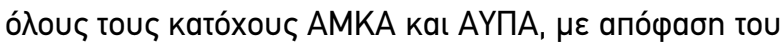

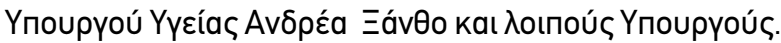

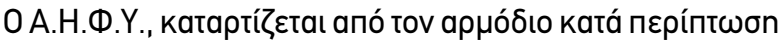

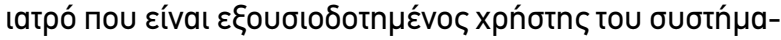

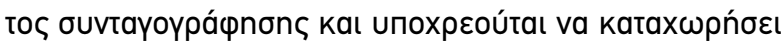

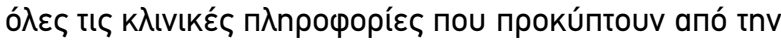

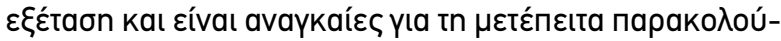

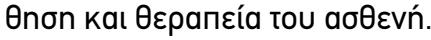

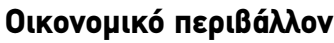

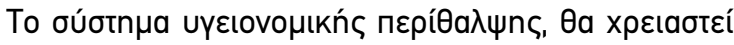

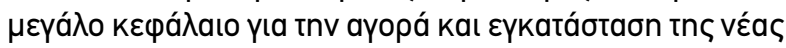

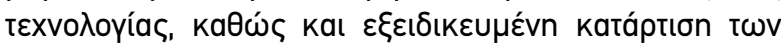

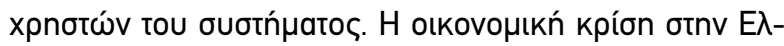

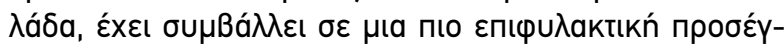

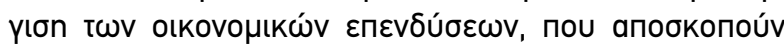

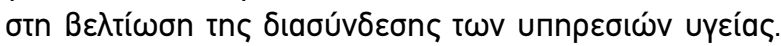

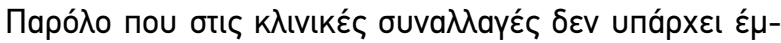

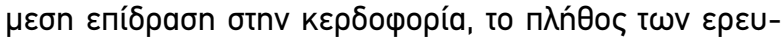

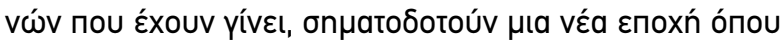

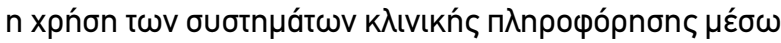

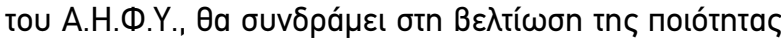

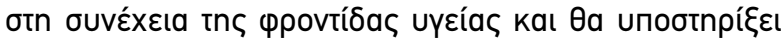

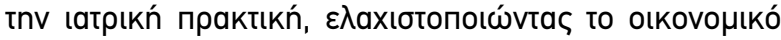

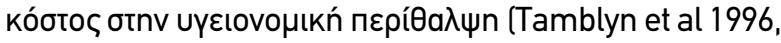
Wagner et al 1996, Bergman et al 2007).

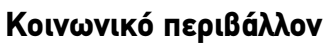

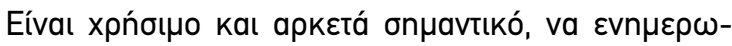

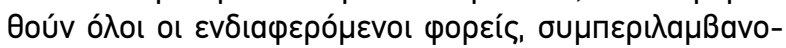

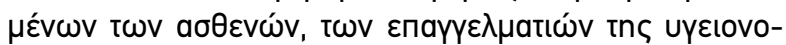

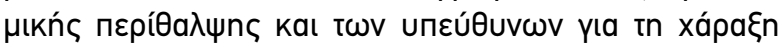

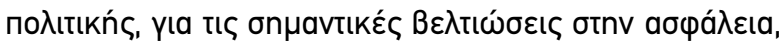

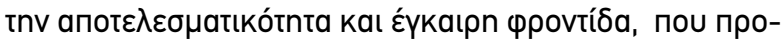

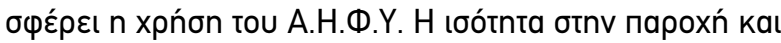

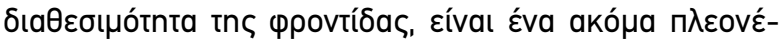

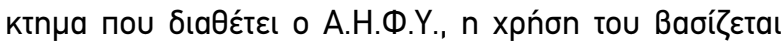

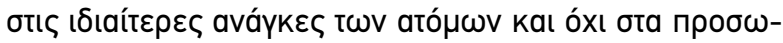

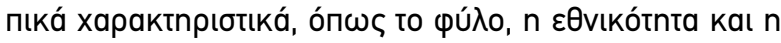

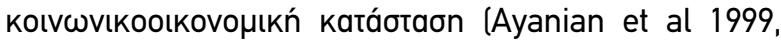
Fiscella et al 2000).

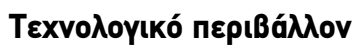

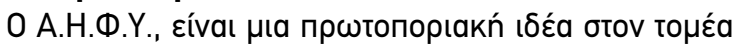

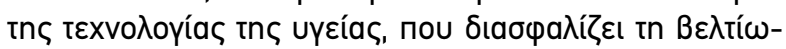

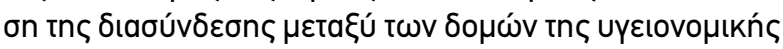

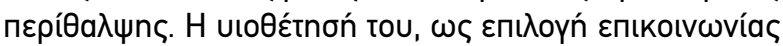

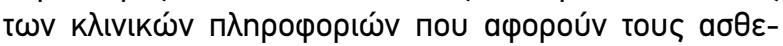

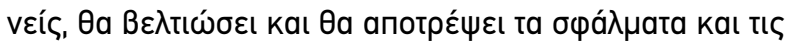

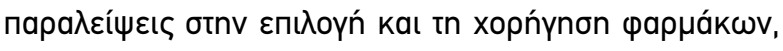

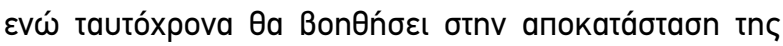

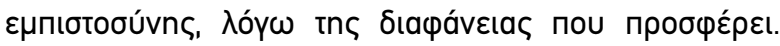

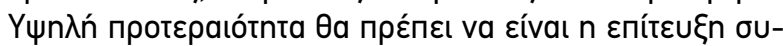

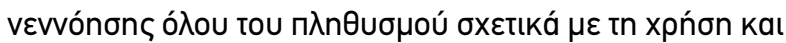

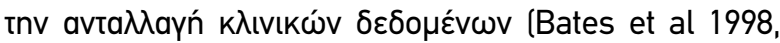
Vanmeerbeek 2004).

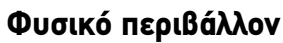

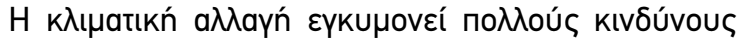

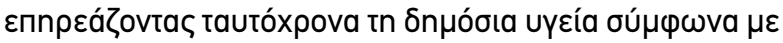

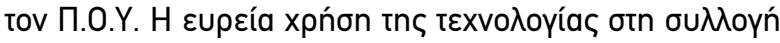

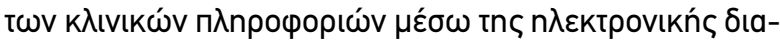

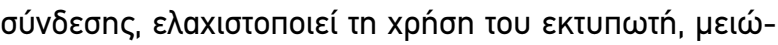

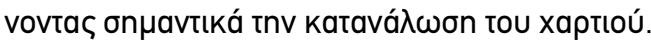

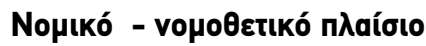

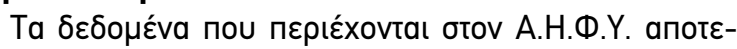

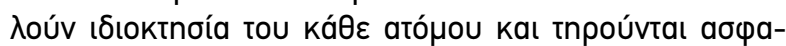

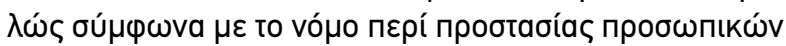

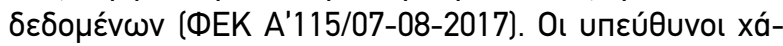

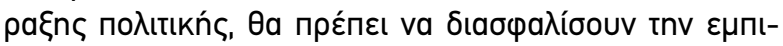

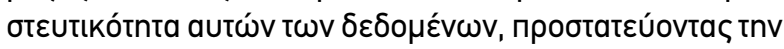

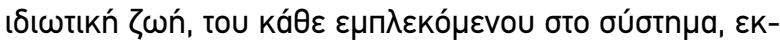

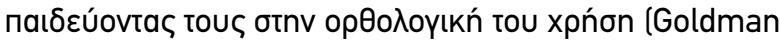

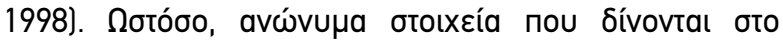

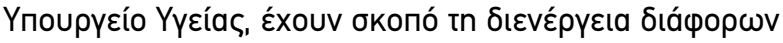

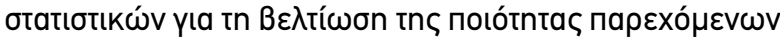

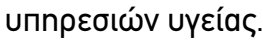

\section{¿YMПEPAгMATA}

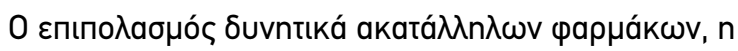

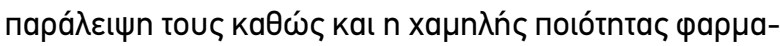

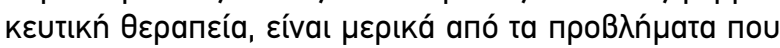

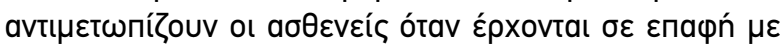

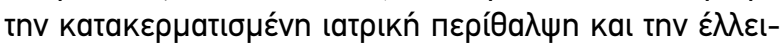

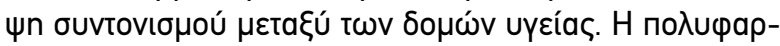

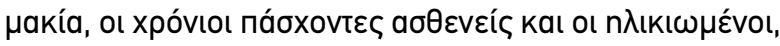

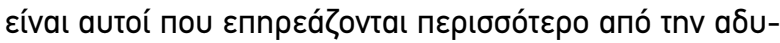

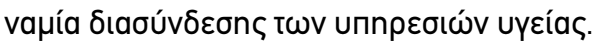

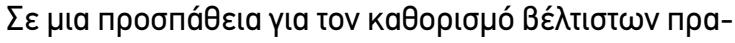

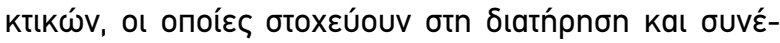

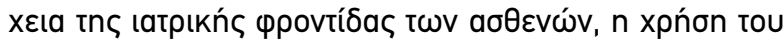

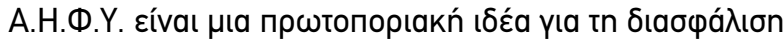

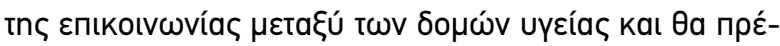

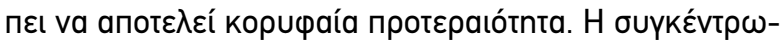

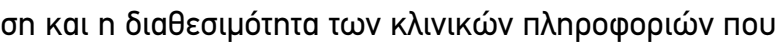

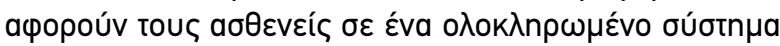

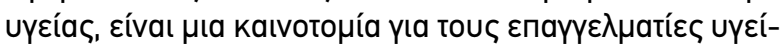

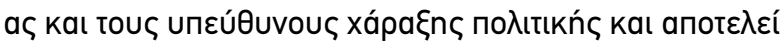

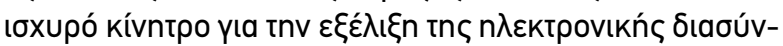

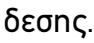

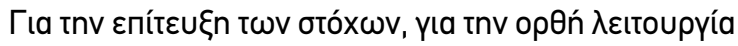




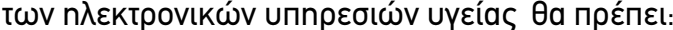

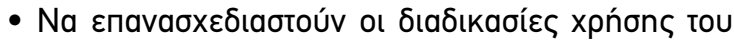

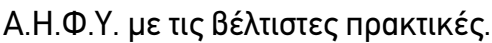

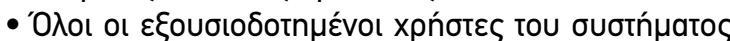

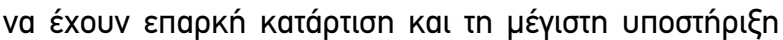

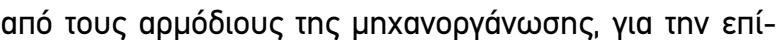

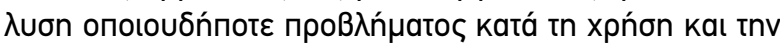

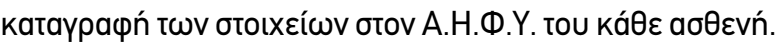

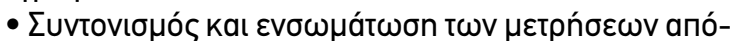

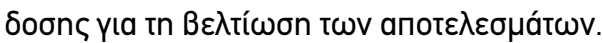

\section{ВIBАIOГРАФIA}

ФEK A'115/07-08-2017, N4486/2017. Taxheaven. URL https://www. taxheaven.gr/laws/law/index/law/830 (accessed 4.22.19).

Abdel-Qader D.H., Cantrill J.A. \& Tully M.P. (2011). Validating reasons for medication discontinuation in electronic patient records at hospital discharge. Journal of Evaluation of Clinical Practice 17:1160-1166

Tremoulet P., Krishnan R., Karavite D., Muthu N., Harkness Regli S., Will A. \& Michel J. (2018). A Heuristic Evaluation to Assess Use of After Visit Summaries for Supporting Continuity of Care. Applied Clinical Informatics 9(3):714-724.

Ayanian J.Z., Weissman J.S., Chasan-Taber S. \& Epstein A.M. (1999). Quality of care by race and gender for congestive heart failure and pneumonia. Medical Care 37:1260-1269.

Bates D.W., Leape L.L., Cullen D.J., Laird N., Petersen L.A., Teich J.M., Burdick E., Hickey M., Kleefield S., Shea B., Vander Vliet M. \& Seger D.L. (1998). Effect of computerized physician order entry and a team intervention on prevention of serious medication errors. JAMA 280:1311-1316

Belleli E., Naccarella L. \& Pirotta M. (2013). Communication at the interface between hospitals and primary care - a general practice audit of hospital discharge summaries. Australian Family Physician 42:886-890.

Bergman A., Olsson J., Carlsten A., Waern M. \& Fastbom J. (2007). Evaluation of the quality of drug therapy among elderly patients in nursing homes. Scandinavian Journal of Primary Healthcare 25 9-14.

Brown K.N., Leigh J.P., Kamran H., Bagshaw S.M., Fowler R.A., Dodek P.M., Turgeon A.F., Forster A.J., Lamontagne F., Soo A. \& Stelfox H.T. (2018). Transfers from intensive care unit to hospital ward: a multicentre textual analysis of physician progress notes. Critical Care 22:19.

Burger C.S. (1997). The use of problem knowledge couplers in a primary care practice. Healthcare Information Management 11:13-26.

Caleres G., Bondesson Å., Midlöv P. \& Modig S. (2018). Elderly at risk in care transitions When discharge summaries are poorly transferred and used -a descriptive study. BMC Health Service Research 18:770.

Callen J., McIntosh J. \& Li, J. (2010). Accuracy of medication documentation in hospital discharge summaries: A retrospective analysis of medication transcription errors in manual and electronic discharge summaries. International Journal Medical Informatics 79:58-64.

Chen Y., Brennan N. \& Magrabi F. (2010). Is email an effective method for hospital discharge communication? A randomized controlled trial to examine delivery of computer-generated discharge summaries by email, fax, post and patient hand delivery. International Journal Medical Informatics 79: 167-172.

Coleman E.A. (2003). Falling through the cracks: challenges and opportunities for improving transitional care for persons with continuous complex care needs. Journal American of Geriatric Society 51:549-555

Coleman E.A., Boult C. for American Geriatrics Society Health Care
Systems Committee (2003). Improving the quality of transitional care for persons with complex care needs. Journal of American Geriatric Society 51:556-557.

Ellenbecker C.H., Frazier S.C. \& Verney S. (2004). Nurses' observations and experiences of problems and adverse effects of medication management in home care. Geriatric Nursing N.Y.N25:164-170.

Fiscella K., Franks P., Gold M.R. \& Clancy C.M. (2000). Inequality in quality: addressing socioeconomic, racial, and ethnic disparities in health care. JAMA 283:2579-2584.

Gandhi T.K. (2005). Fumbled handoffs: one dropped ball after another. Annals of International Medicine 142:352-358.

Goldman J. (1998). Protecting privacy to improve health care. Health Aff. Project Hope 17:47-60.

Grimes T., Delaney T., Duggan C., Kelly J.G. \& Graham I.M. (2008). Survey of medication documentation at hospital discharge: implications for patient safety and continuity of care. Irish Journal of Medical Science 177:93-97.

Johnson A., Guirguis E. \& Grace Y. (2015). Preventing medication errors in transitions of care: A patient case approach. Journal of American Pharmacology Association 55:e264-274; quiz e275-276.

Kripalani S., LeFevre F., Phillips C.O., Williams M.V., Basaviah P. \& Baker D.W. (2007). Deficits in communication and information transfer between hospital-based and primary care physicians: implications for patient safety and continuity of care. JAMA 297:831-841.

LaMantia M.A., Scheunemann L.P., Viera A.J., Busby-Whitehead J. \& Hanson L.C. (2010). Interventions to Improve Transitional Care Between Nursing Homes and Hospitals: A Systematic Review. Journal of American Geriatric Society 58:777-782.

Meyer-Massetti C., Hofstetter V., Hedinger-Grogg B., Meier C.R. \& Guglielmo B.J. (2018). Medication-related problems during transfer from hospital to home care: baseline data from Switzerland. International Journal Clinical Pharmacology 40:16141620.

Meyer-Massetti C., Kaiser E., Hedinger-Grogg B., Luterbacher S. \& Hersberger K. (2012). [Medication safety in the home care setting: error-prone process steps]. Pflege 25:261-269.

Midlöv P., Deierborg E., Holmdahl L., Höglund P. \& Eriksson T. (2008). Clinical outcomes from the use of Medication Report when elderly patients are discharged from hospital. Pharmacology World Science PWS 30:840-845.

Murphy S.F., Lenihan L., Orefuwa F., Colohan G., Hynes I. \& Collins C.G. (2017). Electronic discharge summary and prescription: improving communication between hospital and primary care. Ir. J. Med. Sci. 186:455-459.

Ose D., Baudendistel I., Pohlmann S., Winkler E.C., Kunz A. \& Szecsenyi J. (2017). [Personal health records on the Internet. A narrative review of attitudes, expectations, utilization and effects on health outcomes]. Z. Evidenz Fortbild. Qual. Im Gesundheitswesen 122:921.

Salvi F., Marchetti A., D'Angelo F., Boemi M., Lattanzio F. \& Cherubini A. (2012). Adverse drug events as a cause of hospitalization in older adults. Drug Saf. 35 Suppl 1:29-45. 


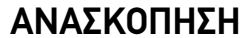

Skoog J., Midlöv P., Beckman A., Sundquist J. \& Halling A. (2015) Indication for pharmacological treatment is often lacking: a cross-sectional study on the quality of drug therapy among the elderly. BMC Geriatry 15:117.

Tamblyn R.M., McLeod P.J., Abrahamowicz M. \& Laprise R. (1996). Do too many cooks spoil the broth? Multiple physician involvement in medical management of elderly patients and potentially inappropriate drug combinations. CMAJ Canadian Medica Association Journal 154:1177-1184.

Tiihonen M., Nykänen I., Ahonen R. \& Hartikainen S. (2016). Discrepancies between in-home interviews and electronic medical records on regularly used drugs among home care clients. Pharmacoepidemiology Drug Saf. 25:100-105.

Vanmeerbeek M. (2004). Exploitation of electronic medical records data in primary health care. Resistances and solutions. Study in eight Walloon health care centres. Studies of Health Technology Informatics 110:42-48.

Viktil K.K., Blix H.S., Eek A.K., Davies M.N., Moger T.A. \& Reikvam A (2012). How are drug regimen changes during hospitalisation handled after discharge: a cohort study. BMJ Open 2.

Wagner E.H., Austin B.T. \& Von Korff M. (1996). Improving outcomes in chronic illness. Management of Care Quality 4:12-25. 


\title{
The Personal Electronic Health Folder and the usefulness of electronic systems to improve health services
}

\author{
Christina Girtsou', Pantelis Stergiannis ${ }^{2,3}$, Theoharis Konstantinidis' ${ }^{1}$, Georgios Martinis ${ }^{1}$ \\ 1. Blood Donation Department, University General Hospital of Alexandroupolis \\ 2. Kifisia's General Oncology Hospital "St. Anargyroi" \\ 3. Hellenic Open University
}

\begin{abstract}
Continuity in patient healthcare shows significant gaps and variations, due to reduced communication between healthcare services. The information transfer to all stages of health structures is often inadequate with many variations, especially at the critical stage after discharge from the hospital, in which patients are usually more vulnerable. The major problem of the lack of interconnection between healthcare services occurs mainly among the elderly, the chronically ill and those who take a lot medication. The main effects of this problem are medication side effects, treatment overlap, poor quality of healthcare and financial costs. Enhancing communication, through the electronic interface and the use of the Personal Electronic Health Folder (P.E.H.F.), which will include the individual details of each patient and information about his clinical status, is aiming at the use of best practices for patient recovery by healthcare providers. The thorough literature review, the strategic analysis PESTEL and the control process, pointed out the need to use the P.E.H.F., to achieve substantial improvements in the quality of patient healthcare. With the practical use of P.E.H.F., the healthcare of all patients will be unquestionably, safe, effective, patient-centered, immediate and fair.
\end{abstract}

Key-words: Healthcare Services, patient, Personal Electronic Health Folder (PEHF) 